\title{
Exceptional Responders in Conservation
}

\author{
Authors: Gerald Post ${ }^{1}$ and Jonas Geldmann ${ }^{2}$ \\ 1 The Veterinary Cancer Center, 129 Glover Ave, Norwalk CT 06850, USA \\ (gpost@VCCHope.com) \\ 2 Conservation Science Group, Department of Zoology, University of Cambridge, Downing St., \\ Cambridge CB2 3EJ, UK (jg794@cam.ac.uk)
}

Person to whom correspondence should be sent: Gerald Post (gpost@vcchope.com)

Article Impact Statement: An exceptional-responder framework may help improve conservation outcomes.

Running title: Exceptional responders in conservation

Keywords: BACI; exceptional responder; evidence-based conservation; impact assessment; outcome; positive deviance; positive outlier

\begin{abstract}
Conservation operates within complex systems with incomplete knowledge of the system and the interventions. This frequently results in the inability to find generally applicable solutions to the threats faced by Earth's vanishing wildlife. One approach used in medicine, and the social sciences has been to develop a deeper understanding of the positive outliers. Where such outliers share similar characteristics, they may be considered: "exceptional responders". Here we present a framework for identifying exceptional responders in conservation. We propose four steps: First, identification of the study system; Second, identification of the response structure; Third, identification of the threshold for exceptionalism; Fourth, identification of commonalities amongst outliers. Evaluating exceptional responders is a method to glean additional information that is often ignored in randomized controlled trials and BACI. Interrogating the contextual factors that contribute to an exceptional outcome allow exceptional responders to become valuable pieces of information leading to unexpected discoveries and novel hypotheses.
\end{abstract}

\section{Introduction}

Human actions are exterminating species at exceptional rates (Pimm et al. 2014), leading to widespread international agreement about the severity of the biodiversity crisis (Convention on

This article has been accepted for publication and undergone full peer review but has not been through the copyediting, typesetting, pagination and proofreading process, which may lead to differences between this version and the Version of Record. Please cite this article as doi: $\underline{10.1111 / \text { cobi.13006. }}$.

This article is protected by copyright. All rights reserved. 
Biological Diversity 2010). Yet, adequate and effective solutions remain elusive, despite a continued increase in conservation efforts (Tittensor et al. 2014).

The discrepancy between increased responses and continued declines has accentuated the need to better understand how to improve the effectiveness of our efforts to halt the loss of biodiversity. One reason for this discrepancy is that conservation is a "wicked problem", operating within complex systems, often with incomplete knowledge of both the system and the interventions (Game et al 2014). This frequently results in the inability to find generally applicable solutions to some of the greatest and clearly identified threats to biodiversity such as poaching, wildlife trafficking, and habitat destruction (Joppa et al. 2016). This has led to a call for evidence-based conservation, emphasizing the use of statically robust experimental design and meta-analysis to identify principal causes and solutions (Sutherland et al. 2004). Such approaches are typically based on evaluating a number of replicated interventions and drawing conclusions based on the mean or median treatment effects (Pullin \& Knight 2001; Sutherland et al. 2004; Centre for Evidence-Based Conservation 2013; O'Leary et al. 2016). Estimates of the average treatment effect are essential, but they can mask important information about the observed heterogeneity (Börner et al. 2016). Without this information, practitioners may fail to improve conservation interventions or abandon interventions that are effective under some circumstances but have low average treatment effect.

Traditionally, heterogeneity is examined empirically through evaluation of the effect of moderators or subgroups. However, this approach is based on a posteriori understanding of what moderators or subgroups to use as well as available data to model the effects. One approach to shedding light on a priori unknown moderators and mechanisms is to develop a deeper understanding, learning from the positive outliers (Baylis et al. 2016). Where such outliers share similar characteristics, they may be considered as "exceptional responders". Examining the differences between the exceptional and non-exceptional responders may identify previously unknown moderators.

In this paper, we will briefly describe how this concept has been applied in oncology and the social sciences. These disciplines have developed more theoretically founded and robust methodologies, to find exceptional responders and glean important information about them and the context in which they exist. We then present a framework for evaluating exceptional responders in conservation. Based on this, we make recommendations about how this concept can be used to obtain information to more effectively save biodiversity and threatened species in particular.

\section{Exceptional Responders in Oncology}

The variability of response to treatment is a hugely important problem in oncology, and drugs that fail to cause regression are often abandoned, even if the drug exhibits profound activity in a small number of patients (Iyer et al. 2012). The US National Cancer Institute's Exceptional

This article is protected by copyright. All rights reserved. 
Responders Initiative (ERI) has developed a framework for classifying these patients and leads the evaluation of exceptional responders in oncology (National Cancer Institute 2015). According to the ERI, patients are classified as exceptional responders if they achieved a complete or durable response after receiving a treatment in which fewer than 10 percent of patients had a complete or durable response (National Cancer Institute 2015). The ERIs work to focus on exceptional responders has been hugely successful. For example, in a trial of 45 people with metastatic bladder cancer treated with everolimus, the primary endpoint of the study: 2-month progression-free survival in $>70 \%$ of the patients, was not met (Milowsky et al. 2013). However, one patient had a near-complete response (Milowsky et al. 2013). When doctors investigated the exceptional responder, they found a mutation in the TSC1 gene (Iyer et al. 2012). When everolimus was tested on additional patients with TSC1-mutant bladder tumors all benefited from the treatment (Figure 1, top).

\section{Exceptional Responders in Social Science}

Solutions to some of the most intractable human problems on the planet, ranging from malnutrition (See Marsh et al. 2004) to human trafficking (Durá \& Singhal 2009) have been found evaluating exceptional responders, often referred to as the positive deviance approach (Spreitzer \& Sonenshein 2004). 'Positive deviants' are organizations or groups that consistently outperform or demonstrate exceptionally high performance metrics (Bradley et al. 2009). The premise of a positive deviance approach is that solutions to intractable problems are often known or practiced by uncommon individuals of the group or community (Marsh et al. 2004). These solutions can be generalized to improve the performance of the entire community as well as other groups (Bradley et al. 2009). Several constructs and definitions have been developed using both statistical and normative approaches (See Spreitzer \& Sonenshein 2004). One of the first and most successful examples of this technique was in the 1990's when researchers reduced the rate of childhood malnutrition by $74 \%$ in children under 3 years of age in Vietnam (Sternin M et al. 1997, 1999). The researchers identified a group of children whose nutritional status was significantly better than the rest of the children and discovered that the mothers of these children were supplementing the diet with tiny shrimps, crabs or snails collected from the rice paddies. This hypotheses was subsequently proven and the practice was adopted by the rest of the village (Marsh et al. 2002) (Figure 1, middle).

\section{An Exceptional Responders Framework for Conservation}

Here, we propose a four-step framework for assessing potential exceptional responders in conservation: 1) Identification of the study system, 2) identification of the response structure, 3 ) identification of the threshold for exceptionalism, and 4) identification of commonalities amongst outliers (Figure 2). To illustrate how the exceptional responder framework can be applied, we use the performance of terrestrial protected areas for large carnivores $(>15 \mathrm{~kg})$ following (Ripple et al. 2014; Ripple et al. 2016; Lindsey et al. 2017).

\section{Identification of the study system}

To understand what makes an exceptional responder, three sets of factors are essential: One, what is the target? Two, what is the treatment? And three, what is the context? As such,

This article is protected by copyright. All rights reserved. 
evaluating exceptional responders share traits with systematic reviews, where tools to address these questions has been developed. A key component of a systematic review is to identify the PICO or PECO (Population, Intervention/Exposure, Comparator, Outcome) elements (Centre for Evidence-Based Conservation 2013). Here, the 'population' is the unit of study to which the intervention will be applied. The 'intervention/exposure' is a management action (i.e. treatment) or a human pressure to which the subject populations are exposed. Here, as in medicine, we define treatment as a complex suite of interventions. The 'comparator' is the control or counterfactual scenario. 'Outcome' is a measure of response in the studied population. Traditional systematic reviews often emphasize average treatment effect, and seek to describe how known contextual factors and differences in study design increase the unexplained variance. In contrast, studies of exceptional responders are particularly interested in the "unknowns" shared by the positive outliers. The exceptional responder system, can reliably generate hypotheses about variance, whether these unknowns are caused by variation in subject/environment or variation in treatment-so long as the type of variance is explicitly stated. We therefore propose a PICOU or PECOU (Population, Intervention/Exposure, Comparator, Outcome, Unknown) assessment to emphasize the important role of exploring how the previously unknown elements shape the variability in responses. In our example, the population would be the large carnivores, while the intervention would be those protected areas in with large carnivores are found; the comparator, a comparable area outside of the protected area; and the outcome would be the difference between carnivore abundance inside these protected areas and outside. The unknown would be potential factors, not included in the original analysis, setting the protected areas with exceptional responses apart from the rest.

\section{Identification of response structure}

By definition, exceptional responders are different from a non-exceptional response. However, how to define an exceptional response will depend on the expectations of the normal response and the type of data being used to measure outcomes. We propose that four different types of exceptional responses exist. One, where the potential outcome is binary: success/failure. Here the exceptional responder is one that succeeds where most fail (e.g. treatment of metastatic bladder cancer). Two, where responses follow a probability distribution (e.g. normal, Poisson, binomial) with a mean and median treatment success, exceptional responders are those "populations" in the extreme right end of the tail. Three, where a posteriori expectation of an outcome exists, an exceptional response is any that exceeds this with some level of magnitude. For example, the International Union for Nature Conservations (IUCNs) Red List has quantitative criteria to identify species under threat: defined as some level of decline for three generations or more than 30 years (International Union for Conservation of Nature 2016). Here, an exceptional responder could be a population of a given species whose population size is increasing or stable for 3 generations or more when the species as a whole is showing threatening declines. Four, in some cases conventional wisdom or established theory dictates specific outcomes. Where such expectations are not meet and outcomes are favorable there is reason to examine these as potential exceptional responders. For example, studies of genetic diversity in many big cat populations showed that the general thinking, linking low genetic diversity to past bottlenecks and increased risk of extirpations, might not be universal (See Amos \& Balmford 2001). In the case of the large carnivores, count data exist (e.g. Walston et al. 2010; Lindsey et al. 2017), which means that a probabilistic distribution can be identified for

This article is protected by copyright. All rights reserved. 
the number of carnivores across protected areas and the difference between inside and outside across protected areas. This can be used to generate an average expected effect of protection as well as identifying some protected areas exceeding this.

\section{Identification of exceptionalism}

Once the nature of the exceptional responders has been determined, a threshold for exceptionalism will need to be defined. Sociology has developed normative approaches focusing on positive deviance as intentional behavior departing from the norm of a reference group (Spreitzer \& Sonenshein 2004). However, we propose that where possible standardized, quantitative, and predefined values should be used. The medical sciences operate with a predefined cut-off (e.g. positive response in $<10 \%$ of patients). For data responses following a probability distribution, a threshold will need to be set. Following general guidelines from probabilistic statistics, any observation more than two standard deviations (S.D.) from the mean or median could be examined as an exceptional responder. However, how to define a threshold will most likely be context specific and should always seek to include all prior knowledge to ensure that heterogeneity from already known sources are not attributed to exceptionalism. For the large carnivores, this could involve modelling how the difference between abundance inside protected areas and outside depends upon contextual factors such as protected area size and location to identify those protected areas where abundance was higher than two S.D.'s of the model expectations.

\section{Identification of commonalities}

The final step is to interrogate the potential exceptional responders for commonalities. While a single outlier might still be of interest, as a case study, common properties and shared values of multiple positive outliers will help to test the general validity of the exceptional response. In most cases identification of properties shared by the exceptional responders will need additional experimental or quasi-experimental testing to understand the underlying mechanisms behind the observed response. For this, interdisciplinary teams are tremendously valuable as each discipline may identify different commonalities. We see exceptional responder analysis, a hypothesis generating system, as a method to allow people from various disciplines to delve into the context and identify possible commonalities. Thus, a priori defining what to look for will be near impossible, and identifying potential exceptional responders will require a detailed understanding of known variation to identify previously unknown components contributing to the variation. However, the use of a Theory of Change architecture can focus inter- and multi-disciplinary teams (De Silva et al. 2014) on commonalities that fit within the study parameters. For the large carnivores, this final step involves having an interdisciplinary team interrogate the commonalities shard by the exceptional responders identified. Experimental or quasi-experimental studies could then be designed to test hypotheses generated by the interdisciplinary team.

\section{Examples of exceptional responders in conservation}

We know of only very few examples in the conservation literature that have used traits shared by the positive outliers to illuminate novel moderators. In one study evaluating 42 tiger

This article is protected by copyright. All rights reserved. 
reserves in India, Post and Pandav (2013) found that seven reserves succeeded above expectations. Exploring the successful reserves, the researchers identified the presence of specifically dedicated individuals "conservation champions" as a common trait shared by most of the successful reserves (Post \& Pandav 2013). Perhaps the best example came from a recent study on coral reef health, across 2,500 reefs worldwide, where the authors identified 'Bright spots' and 'Dark spots', as coral patches that were substantially better or worse than expected based upon Bayesian hierarchical modelling (Cinner et al. 2016). Here Cinner et al. (2016) used a suite of known environmental and socio-economic variables to understand the performance of protected areas. Following this they defined 'bright spots' and 'dark spots' as two standard deviations (S.D.) from the predicted outcome in each location, not the mean observed response. Modeling the heterogeneity showed, surprisingly, that these bright spots were predominantly located in areas with high human populations and where fishing occurred (Cinner et al. 2016). However, by surveying local experts the authors revealed that bright spots also were more likely to have high levels of engagement with local communities and the presence of sociocultural governance institutions (Cinner et al. 2016) (Figure 1, bottom). Interrogating the outliers allowed Cinner et al. (2016) to identify commonalities that were previously unknown. Thus, while the factors identified, after the fact, might not seem exceptional, they were only discovered by understanding what set the exceptional response apart from the expected.

\section{Discussion}

There is a growing interest by conservation scientists, practitioners, and policy makers in evidence of what interventions result in successful conservation outcomes (Sutherland et al. 2004; Kapos et al. 2008). Likewise, donors are increasingly interested in assessing and understanding their programmatic effectiveness (Redford \& Taber 2000; Craigie et al. 2015). However, despite increased attention on improving conservation responses our understanding of what works and why is still limited (Ferraro \& Hanauer 2014). Analyzing the exceptional responders and their context may uncover hypotheses about why certain conservation interventions are successful and others fail. The exceptional responder framework is a hypothesis generating approach. Thus, to ensure that chance outliers or an erroneous result are not falsely interpreted as exceptional responders, experimental or quasi-experimental studies should be completed prior to any information gleaned through this approach being used to inform policy.

Studying the exceptional responders in conservation is not a replacement for experimental design or using Before-After Control-Intervention (BACI) designed experiments. And we do not dispute that the randomized controlled clinical trial is the gold standard for providing evidence. Rather, evaluation of exceptional responders is a method to glean additional information that is not captured with these more conventional techniques. Exceptional responder evaluations can help identify potential moderators of heterogeneity, which can then be further scrutinized and analyzed to ensure that they are not due to chance events. For example, using the results of the exceptional responder analysis to identify new cases, similar to the exceptional outlier, and then ensuring that the observed treatment effect persists and can be explained. This would be similar

This article is protected by copyright. All rights reserved. 
to the bladder tumor example where additional patients with mutations in the TSC1 gene were examined (Milowsky 2013). Thus, where traditional impact evaluations look for what works in a broad swath of the population/programs (Mascia et al. 2014), exceptional responders, in contrast evaluates the context of the outliers with the aim of giving us clearer signals for hypothesis generation and applying the knowledge to a larger subset. This allows for exploring variables that might be insignificant in explaining general patterns, but are shared by the positive outliers. Knowledge from evaluations of exceptional responders can thus be used to interrogate moderators not previously considered in the treatment design.

One of the challenges in conservation studies, unlike medicine, is that BACI or true experimental approaches are very difficult, sometime impossible, to carry out, and hence are rare. In addition, the sample size available for conservation scientists to pursue the exceptional responder approach is much smaller than in medicine. However, as the bladder cancer example (Milowsky et al 2013) demonstrates, as few as 20-50 cases can be enough to both identify outliers and develop useful hypotheses for further testing. Thus, analysis of exceptional responders is not different from other statistical approaches, where the adequate sample sizes depends on the experimental setup, ability to exclude confounding factors and the expected effect size of the moderator in question.

It is not uncommon in conservation science for successful examples to be studied in detail as case studies. The difference between learning from a single case and exceptional responder analysis, is that the latter is based on examining commonalities within a set of samples. Thus, while isolated case studies have tremendous value, and the lessons learned can be transferred to similar situations elsewhere, they are often difficult to use as a basis for examining exceptional responders because individual cases often have no easily referenced sample set from which case studies are drawn. This situation may very well change as broad initiatives such as Conservation Evidence (Conservation Evidence 2017) and groups of researchers (e.g. Bauer et al. 2015; Cinner et al. 2016) continue to generate and expand databases assembling case-studies to evaluate conservation effectiveness.

One of the challenges of the "exceptional responder" approach is that the evidence gleaned is often suggestive and may be simply due to sampling variability in experimental or observational studies or hidden bias (e.g. selection bias) in observational designs. There is also the problem that these outliers may be due largely to chance events and regression to the mean may act in future years. Thus, the causal nature of the exceptional response is difficult to prove without further experimental or quasi-experimental studies. Rather, the exceptional responder approach in conservation generates hypotheses.

Interdisciplinary knowledge and skills are extremely valuable in the exceptional responder approach. Social scientists' expertise greatly facilitates the evaluation of socio-economic context

This article is protected by copyright. All rights reserved. 
and designing experimental or quasi-experimental studies to test the hypotheses generated from the exceptional responder approach (Cinner et al. 2016). The exceptional responder approach can also help bridge the knowledge action gap, through facilitating that new information is not only obtained, but also transmitted and used in practical settings. Evaluating exceptional responders often involve practitioners and local community members to fully understand the underlying reasons of what sets the outlier apart from the average. The inclusion and recognition of the importance of indigenous and local knowledge through the inclusion of multiple stakeholders in the process can help increase the legitimacy of the information produced and thus increase the likelihood of knowledge uptake (Cook et al. 2013). Indeed, legitimacy is cited as the key determinant in linking knowledge to action (Rowe \& Lee 2012) and the value of working with people trained as social scientists, economists, behavioral economists, and others-both in the early design phase and throughout the study is well documented in the conservation literature (Bennett et al. 2016). Our proposed methodology may allow outcomes to be evaluated on similar scales to management actions. The exceptional responder approach can be employed at both small (e.g. childhood nutrition in a few villages (Marsh et al 2002) or tiger conservation in a single region (Post and Pandav 2013)) and large scales (e.g. Cinner et al 2016). Scale-matching is one of the core principles to sustainable governance of protected areas (Costanza et al 1999). In addition, our methodology offers an easy and transparent approach to identifying potential outliers.

We have focused on the positive outliers. However, conservation scientists, practitioners and policy makers can also use the 'exceptional responders' technique to compare and contrast outliers in both directions - exceptional and failed responders to identify differences between them (Post \& Pandav 2013; Cinner et al. 2016). Such negative outliers may be equally informative in identifying traits that should be avoided when setting up conservation programs.

Identifying the exceptional responders when there is poor monitoring and reporting of outcomes in the literature is a challenge. Further, a problem in conservation is the lack of uniform measures of effectiveness which makes it difficult to compare and contrast results across studies in a comparable manner (Geldmann et al. 2013). For these and other reasons, monitoring and outcome reporting are hugely important for conservation (Nichols \& Williams 2006). In addition, because of publication bias (Dickersin 1990), failed or poor responders are often not documented and reported, making the identification of outliers difficult (Prasad \& Vandross 2015). Thus, future conservation research should report the entire range of results or responses to interventions or treatments. The significance of this type of evaluation in terms of scope and applicability is often unknown until the factors contributing to the exceptional response are discerned. Another key challenge with this approach is determining whether the exceptional response is due to the treatment and context together, versus the unique aspects of the context alone (Prasad \& Vandross 2015). Identifying exceptional responders does not guarantee that the factors contributing to its success can be easily replicated, however, the information obtained about how the treatment and contextual elements interact is extremely valuable (Subbiah \& Subbiah 2015).

This article is protected by copyright. All rights reserved. 


\section{Conclusion}

Information about what conservation interventions work in which contexts is sorely needed. This evidence also must be credibly generated and transmitted so that adoption by practitioners and policy makers is likely. The evaluation of exceptional responders in the social sciences enabled seemingly intractable problems to be solved and in medicine this methodology uncovered contextual elements that were critical for treatments to be successful. The problems faced by conservation scientists share many of the same features as those faced by medical professionals and social scientists; many are "wicked" or seemingly intractable problems, and conservation exists within a complex bio-geo-socio-economic framework where context is hugely important. Utilizing the methodology discussed in this paper will allow scientists to better discern what conservation interventions should work in which contextual settings. This knowledge will be complementary to the evidence gleaned from the more traditional methods of randomized controlled trials and BACI studies.

\section{Acknowledgments}

We thank S. L. Pimm, K. Redford, J. Cinner, and P. Ferraro for valuable advice, discussions, and comments on the manuscript. We thank W. Sutherland and A. Balmford for valuable advice and discussions. Financial support for J.G. comes from VILLUM FUNDEN (VKR023371).

\section{References}

Amos W, Balmford A. 2001. When does conservation genetics matter? Heredity 87:257-265.

Bauer H, Chapron G, Nowell K, Henschel P, Funston P, Hunter LTB, Macdonald DW, Packer C. 2015. Lion (Panthera leo) populations are declining rapidly across Africa, except in intensively managed areas. Proceedings of the National Academy of Sciences.

Baylis K, Honey-Rosés J, Börner J, Corbera E, Ezzine-de-Blas D, Ferraro PJ, Lapeyre R, Persson UM, Pfaff A, Wunder S. 2016. Mainstreaming Impact Evaluation in Nature Conservation. Conservation Letters 9:58-64.

Bennett NJ, et al. 2016. Mainstreaming the social sciences in conservation. Conservation Biology:n/an/a.

Börner J, Baylis K, Corbera E, Ezzine-de-Blas D, Ferraro PJ, Honey-Rosés J, Lapeyre R, Persson UM, Wunder S. 2016. Emerging Evidence on the Effectiveness of Tropical Forest Conservation. PLOS ONE 11:e0159152.

Bradley EH, Curry LA, Ramanadhan S, Rowe L, Nembhard IM, Krumholz HM. 2009. Research in action: using positive deviance to improve quality of health care. Implementation Science 4:25.

Centre for Evidence-Based Conservation. 2013. Guidelines for Systematic Review in Environmental Management. Version 4.2. Conservation CfE-B, Bangor University, UK.

This article is protected by copyright. All rights reserved. 
Cinner JE, et al. 2016. Bright spots among the world's coral reefs. Nature 535:416-419.

Conservation Evidence. 2017. conservationevidence.com, Cambridge, UK. Available from http://www.conservationevidence.com/ 2017).

Convention on Biological Diversity. 2010. Strategic Plan for Biodiversity 2011-2020 - COP 10, decision X/2, Available from http://www.cbd.int/decision/cop/?id=12268 (accessed 25/4 2013 2013).

Cook CN, Possingham HP, Fuller RA. 2013. Contribution of Systematic Reviews to Management Decisions. Conservation Biology 27:902-915.

Craigie ID, Barnes MD, Geldmann J, Woodley S. 2015. International funding agencies: potential leaders of impact evaluation in protected areas? Philosophical Transactions of the Royal Society of London B: Biological Sciences 370:20140283.

De Silva MJ, Breuer E, Lee L, Asher L, Chowdhary N, Lund C, Patel V. 2014. Theory of Change: a theory-driven approach to enhance the Medical Research Council's framework for complex interventions. Trials 15:267.

Dickersin K. 1990. The Existence of Publication Bias and Risk Factors for Its Occurrence. JAMA 263:1385-1389.

Durá L, Singhal A. 2009. Utilizing a Positive Deviance Approach to Reduce Girls' Trafficking in Indonesia. Journal of Creative Communications 4:1-17.

Ferraro PJ, Hanauer MM. 2014. Advances in Measuring the Environmental and Social Impacts of Environmental Programs. Pages 495-517 in Gadgil A, and Liverman DM, editors. Annual Review of Environment and Resources, Vol 39.

Geldmann J, Barnes M, Coad L, Craigie ID, Hockings M, Burgess ND. 2013. Effectiveness of terrestrial protected areas in reducing habitat loss and population declines Biological Conservation 161:230-238.

International Union for Conservation of Nature. 2016. The IUCN Red List of Threatened Species, Available from http://www.iucnredlist.org 2016).

Iyer G, et al. 2012. Genome Sequencing Identifies a Basis for Everolimus Sensitivity. Science 338:221221.

Joppa LN, et al. 2016. Filling in biodiversity threat gaps. Science 352:416-418.

Kapos V, et al. 2008. Calibrating conservation: new tools for measuring success. Conservation Letters 1:155-164.

Lindsey PA, et al. 2017. The performance of African protected areas for lions and their prey. Biological Conservation 209:137-149.

Marsh DR, Pachón H, Schroeder DG, Ha TT, Dearden K, Lang TT, Hien ND, Tuan DA, Thach TD, Claussenius D. 2002. Design of a Prospective, Randomized Evaluation of an Integrated Nutrition Program in Rural Viet Nam. Food and Nutrition Bulletin 23:34-44.

Marsh DR, Schroeder DG, Dearden KA, Sternin J, Sternin M. 2004. The power of positive deviance. British Medical Journal 329:1177-1179.

This article is protected by copyright. All rights reserved. 
Mascia MB, Pailler S, Thieme ML, Rowe A, Bottrill MC, Danielsen F, Geldmann J, Naidoo R, Pullin AS, Burgess ND. 2014. Commonalities and complementarities among approaches to conservation monitoring and evaluation. Biological Conservation 169:258-267.

Milowsky MI, et al. 2013. Phase II study of everolimus in metastatic urothelial cancer. BJU International 112:462-470.

National Cancer Institute. 2015. Exceptional Responders Initiative, Available from https://www.cancer.gov/news-events/press-releases/2014/ExceptionalRespondersQandA (accessed 17-10-2016 2016).

Nichols JD, Williams BK. 2006. Monitoring for conservation. Trends in Ecology \& Evolution 21:668673.

O'Leary BC, Kvist K, Bayliss HR, Derroire G, Healey JR, Hughes K, Kleinschroth F, Sciberras M, Woodcock P, Pullin AS. 2016. The reliability of evidence review methodology in environmental science and conservation. Environmental Science \& Policy 64:75-82.

Pimm SL, Jenkins CN, Abell R, Brooks TM, Gittleman JL, Joppa LN, Raven PH, Roberts CM, Sexton JO. 2014. The biodiversity of species and their rates of extinction, distribution, and protection. Science 344.

Post G, Pandav B. 2013. Comparative evaluation of tiger reserves in India. Biodiversity and Conservation 22:2785-2794.

Prasad V, Vandross A. 2015. Characteristics of Exceptional or Super Responders to Cancer Drugs. Mayo Clinic Proceedings 90:1639-1649.

Pullin AS, Knight TM. 2001. Effectiveness in conservation practice: Pointers from medicine and public health. Conservation Biology 15:50-54.

Redford KH, Taber A. 2000. Writing the Wrongs: Developing a Safe-Fail Culture in Conservation. Conservation Biology 14:1567-1568.

Ripple WJ, et al. 2016. Saving the World's Terrestrial Megafauna. BioScience 66:807-812.

Ripple WJ, et al. 2014. Status and Ecological Effects of the World's Largest Carnivores. Science 343.

Rowe A, Lee KN. 2012. Linking Knowledge with Action: an approach to philanthropic funding of science for conservation. Foundation DLP, Los Altos, CA, USA.

Spreitzer GM, Sonenshein S. 2004. Toward the construct definition of positive deviance. American Behavioral Scientist 47:828-847.

Sternin M, Sternin J, Marsh D. Rapid. 1997. sustained childhood malnutrition alleviation through a "positive deviance" approach in rural Vietnam: preliminary findings. Pages 19-21 in Keeley E BB, Wollinka O, Bashir N, editor. The hearth nutrition model: applications in Haiti, Vietnam, and Bangladesh, Report of a Technical Meeting at World Relief Corporation Wheaton, IL BASICS, Arlington, USA.

Sternin M, Sternin J, Marsh D. Rapid. 1999. Scaling up a poverty alleviation and nutrition program in Viet Nam in Marchione T, editor. Scaling up, scaling down: capacities for overcoming malnutrition in developing countries. Gordon and Breach, Amsterdam, the Netherlands.

This article is protected by copyright. All rights reserved. 
Subbiah IM, Subbiah V. 2015. Exceptional responders: in search of the science behind the miracle cancer cures. Future Oncology 11:1-4.

Sutherland WJ, Pullin AS, Dolman PM, Knight TM. 2004. The need for evidence-based conservation. Trends in Ecology \& Evolution 19:305-308.

Tittensor DP, et al. 2014. A mid-term analysis of progress toward international biodiversity targets. Science 346:241-244.

Walston J, et al. 2010. Bringing the Tiger Back from the Brink-The Six Percent Solution. PLOS Biology 8:e1000485.

\section{Figure legends:}

Figure 1. Examples of the exceptional responder technique used in oncology (top), nutritional studies (middle), and coral-reef conservation (bottom). Charts on the right describes the outcome of studying exceptional responders where either a novel treatment is discovered for a subgroup (top) or a general improvement is observed across a population by implementing approaches only previously known by a sub-group (middle and bottom).
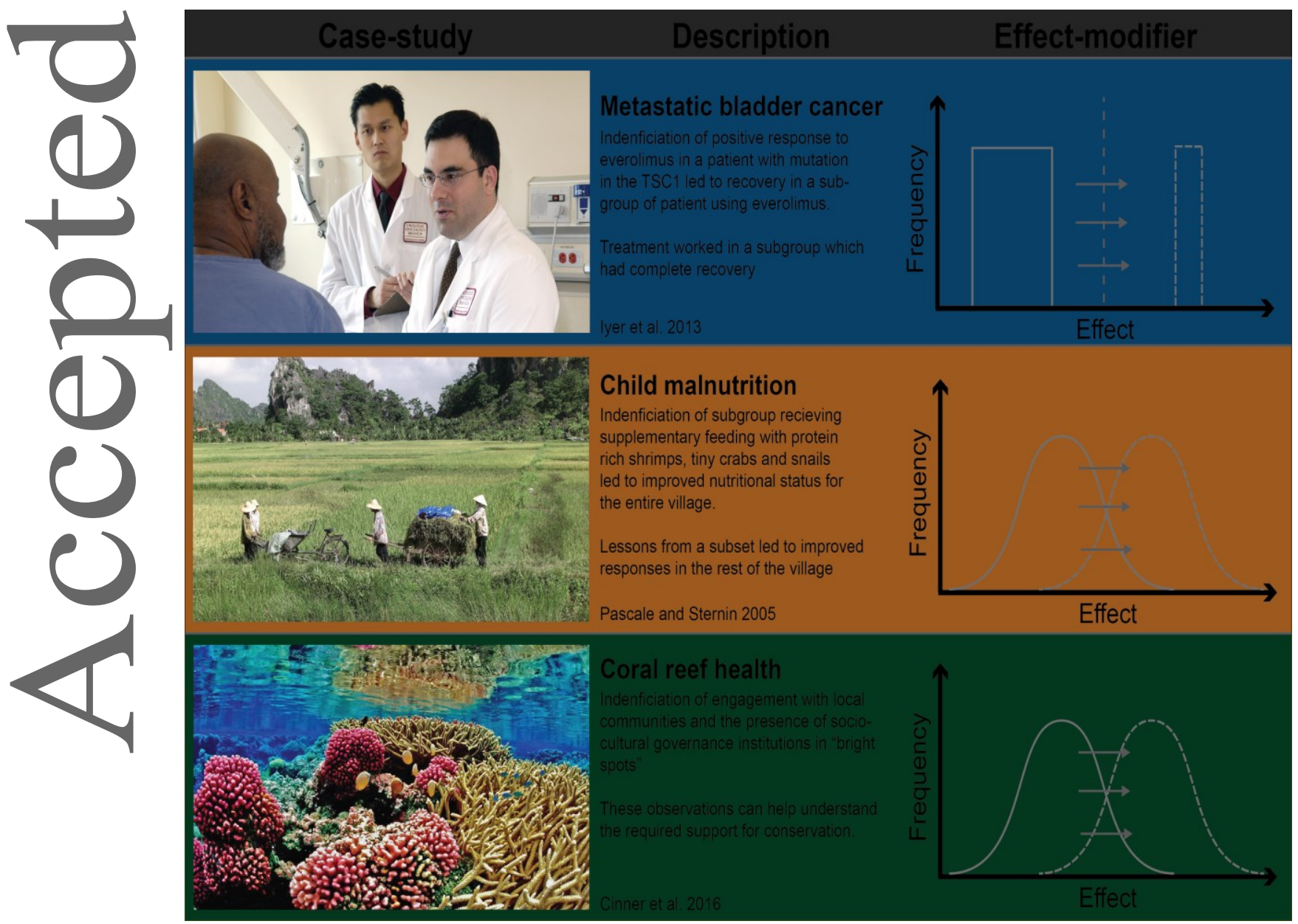

This article is protected by copyright. All rights reserved. 
Figure 2. Exceptional responder framework, outlining the four steps from identification of study system, via identification of response structure and exceptionalism to identification of commonalities amongst positive outliers. The Outcome of the identification will usually either be an improved average response through the identification of a novel mechanism (bottom left) or a novel approach for a sub-group or the entire population (bottom right).
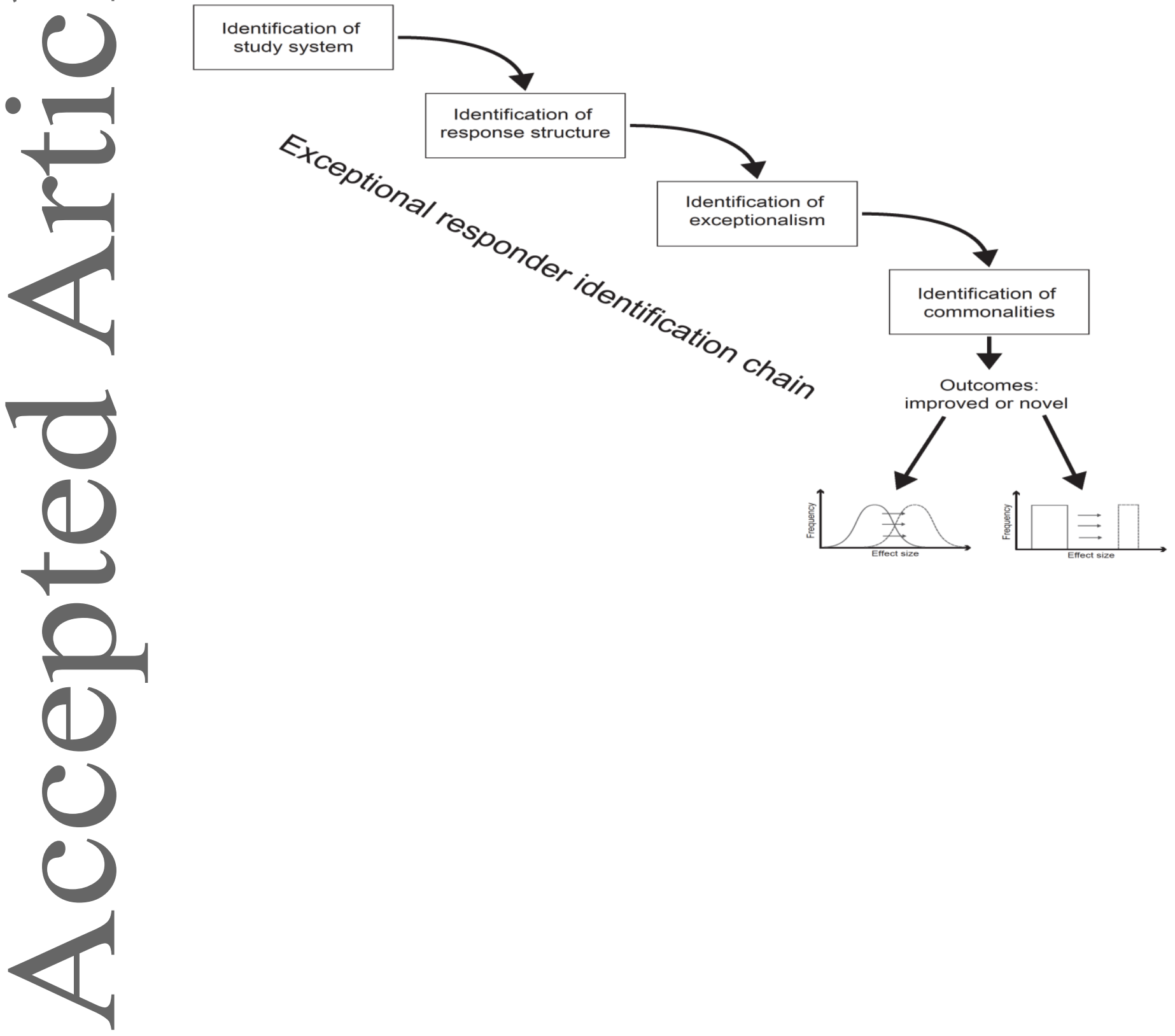

This article is protected by copyright. All rights reserved. 